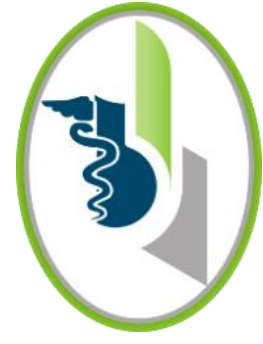

ACCESO 2 ABIERTO

Para citaciones: Burbano, A., Ayola, C. y Ramos, E. (2019). Adherencia farmacológica en relación con funcionalidad familiar en el tratamiento ambulatorio de pacientes con esquizofrenia, depresión y trastorno bipolar. Revista Ciencias Biomédicas, $8(2), 40-50$

Recibido: 16 de mayo de 2019 Aprobado: 23 de junio de 2019

Autor de correspondencia: Adriana Lucia Burbano López md.psiquiatria.positiva@gmail.com

Editor: Inés Benedetti. Universidad de Cartagena-Colombia.

Copyright: (C) 2019. Burbano, A., Ayola, C. y Ramos, E. Este es un artículo de acceso abierto, distribuido bajo los términos de la licencia https://creativecommons.org/licenses/by-nc-

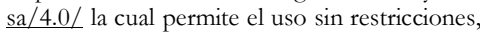
distribución y reproducción en cualquier medio, siempre y cuando el original, el autor y la fuente sean acreditados.

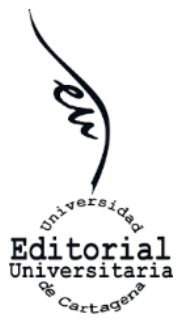

\section{Adherencia farmacológica en relación con funcionalidad familiar en el tratamiento ambulatorio de pacientes con esquizofrenia, depresión y trastorno bipolar}

\author{
Pharmacological adherence in relation to family functionality in \\ outpatient treatment of patients with schizophrenia, depression \\ and bipolar disorder.
}

Burbano López Adriana Lucía ${ }^{1}$, Ayola Gómez Christian ${ }^{1}$, Ramos Clason Enrique Carlos ${ }^{2}$

${ }^{1}$ Sección de Psiquiatría. Facultad de Medicina. Universidad de Cartagena-Colombia
${ }^{2}$ Departamento de Investigaciones, Facultad de Medicina. Universidad de Cartagena-Colombia

\section{RESUMEN}

Introducción: la escasez de estudios epidemiológicos acerca de las características clínicas y farmacológicas de pacientes con enfermedades mentales manejados de forma ambulatoria a nivel nacional y local, acarrea un insuficiente conocimiento médico para realizar un seguimiento eficaz en el manejo de patologías tan comunes en psiquiatría como la esquizofrenia, el trastorno bipolar y la depresión.

Objetivo: caracterizar clínica y farmacológicamente a pacientes ambulatorios manejados en una clínica psiquiatrica en Cartagena, e identificar si hay relación entre la adherencia farmacológica, tipo de diagnóstico y grado de disfunción de familiar.

Métodos: estudio descriptivo de corte transversal con 160 pacientes ambulatorios mayores de 18 años y menores de 65 años diagnosticados con esquizofrenia, trastorno bipolar y depresión, a quienes se les aplicó un cuestionario de características clínico-farmacológicas, el cuestionario de Morisky-Green y el Test de Apgar Familiar.

Resultados: el 68,1\% de los encuestados refirieron conocer su diagnóstico psiquiátrico, el principal diagnóstico fue el trastorno afectivo bipolar $(35,6 \%)$, seguido de la esquizofrenia $(34,4 \%)$ La aplicación del APGAR familiar mostró una frecuencia de familia disfuncional en el 36,3\%, La escala de Morisky Green mostró una no adherencia total al tratamiento de 53,8\%. Al comparar la frecuencia de diagnósticos, funcionalidad familiar, estratificado, por grupos de pacientes con y sin adherencia al tratamiento, no se observaron diferencias estadísticamente significativas.

Conclusiones: Se encontró un alto porcentaje de no adherencia farmacológica y la gran mayoría reportó tener familias funcionales. Sin embargo, no se encontró relación estadísticamente significativa entre la adherencia al tratamiento, el tipo de diagnóstico ni el grado de disfunción familiar.

Palabras Clave: Esquizofrenia; depresión; cumplimiento y adherencia al tratamiento farmacológico; pacientes ambulatorios; familia. 


\begin{abstract}
Introduction: the scarcity of epidemiological studies about the clinical and pharmacological characteristics of patients with mental illnesses managed on an outpatient basis at the national and local level, leads to insufficient medical knowledge to effectively follow up on the management of such common pathologies in psychiatry as schizophrenia, bipolar disorder and depression.
\end{abstract}

Objective: to characterize clinically and pharmacologically outpatients managed in a psychiatric clinic in Cartagena, and to identify whether there is a relationship between pharmacological adherence, type of diagnosis, and degree of family dysfunction.

Methods: descriptive cross-sectional study with 160 outpatients over 18 and under 65 years of age diagnosed with schizophrenia, bipolar disorder, and depression, to whom a questionnaire of clinical-pharmacological characteristics, the MoriskyGreen questionnaire, and the Family Apgar Test were applied.

Results: $68.1 \%$ of those surveyed reported knowing their psychiatric diagnosis, the main diagnosis being bipolar affective disorder (35.6\%), followed by schizophrenia (34.4\%). The application of the Family APGAR showed a dysfunctional family frequency in $36.3 \%$, and the Morisky-Green scale showed a total non-adherence to treatment of $53.8 \%$. When comparing the frequency of diagnosis, family functionality, and stratification by groups of patients with and without adherence to treatment, no statistically significant differences were observed.

Conclusion: A high percentage of pharmacological nonadherence was found, and the great majority reported having functional families. However, no statistically significant relationship was found between adherence to treatment, type of diagnosis, or degree of family dysfunction.

Key Words: Schizophrenia; depression; treatment adherence and compliance; outpatients; family.

\section{INTRODUCCIÓN}

La esquizofrenia y los trastornos del estado de ánimo hacen parte de las enfermedades mentales más diagnosticadas en la práctica clínica (1). Son consideradas enfermedades crónicas que al no ser tratadas de la manera adecuada conducen a resultados sanitarios y educativos bajos, poca participación en la economía y altas tasas de pobreza (2).

Los estudios estadísticos evidencian un incremento de las prevalencias en los últimos años, con datos actuales a nivel mundial en esquizofrenia de aproximadamente $1 \%$ (3), en el trastorno bipolar de $0.3-1.5 \%$ (4),(5), y la depresión alrededor de $6.7 \%$
(6). En Colombia, en un estudio realizado en el 2017 en el que se analizaron síntomas sugestivos de trastorno mental en adultos sin diagnostico psiquiátrico, se concluyó que el $80.2 \%$ manifestaban de uno a tres síntomas depresivos y el $6.7 \%$ tenían indicadores de psicosis (7).

En Cartagena, de 1.720 casos de patologías mentales que se atendieron durante el año 2016, los trastornos del estado de ánimo reportaron el mayor porcentaje de casos con un 45,3\%, seguido de los trastornos psicóticos con un 42,5\%, el tercer lugar le correspondió al consumo de sustancias psicoactivas con un 3,2\%. Dentro de los trastornos del estado de ánimo se encuentran los transtornos bipolares, y los episodios depresivos (8). 
Estos trastornos requieren para su estabilidad una atención médica continua con tratamiento farmacológico unido con otras medidas terapéuticas, debido a que tienden a ser severos con ciclos de recaídas y remisiones (9). Sin embargo, la frecuencia de recaídas en pacientes ambulatorios no disminuye tanto como es de esperar (10). Uno de los factores que más ha influido en que esto ocurra es la falta de cumplimento o adherencia terapéutica en estos pacientes, debido a que generalmente, tienen una gran dificultad para seguir un régimen de medicación, pero también tienen el mayor potencial para beneficiarse de este (11) (12). En la práctica clínica hasta un $55 \%$ de pacientes con esquizofrenia que no toman los medicamentos antipsicóticos formulados recaerá en un lapso de un año, en comparación con solo el $14 \%$ de los pacientes que se adhieren a la medicación (13). Del mismo modo, se ha descrito que el $60 \%$ de los pacientes admitidos con manía no habían podido adherirse a la medicación formulada en el mes anterior a dicha hospitalización (14). En la depresión, las tasas de recaídas al año son del $80 \%$ en pacientes que no toman las drogas antidepresivas y del $30 \%$ para los que si las toman (13)(15). La Organización Mundial de la salud -OMS- definió en el 2001 el cumplimiento terapéutico como la magnitud con que el paciente sigue las instrucciones médicas, incorporando hasta hace algunas décadas el término adherencia, que se utiliza para hacer referencia a un compromiso voluntario y colaborador por parte del paciente con el objetivo mutuo de conseguir el resultado preventivo o terapéutico deseado (16)(17).

En el caso de la esquizofrenia y el trastorno bipolar, la mayoría de los estudios a nivel mundial reportan cifras de mala adherencia a los medicamentos recetados en torno al 51\%-70\% (18)(19). En cuanto a la depresión las cifras tampoco son alentadoras, reportando que entre el $40 \%$ y el $70 \%$ de los pacientes no se adhieren a las terapias antidepresivas (20)(21).

Hasta el momento en la región hay pocos estudios epidemiológicos disponibles que describan las características clínicas y farmacológicas de pacientes ambulatorios con diagnósticos psiquiátricos frecuentes (trastornos del estado de ánimo/esquizofrenia), por lo cual los médicos no tenemos referencias basadas en la evidencia para brindar la mejor atención a nuestros pacientes, por lo tanto, es pertinente realizar una aproximación caracterológica sobre este tema enfocada en nuestra población local. Por esto, el presente estudio tuvo como objetivo caracterizar clínica y farmacológicamente a pacientes ambulatorios con esquizofrenia, trastorno afectivo bipolar y depresión en una clínica de salud mental en la ciudad de Cartagena de Indias, Colombia, e identificar si existe relación entre la adherencia al tratamiento farmacológico en estos pacientes por tipo de diagnóstico y por grado de disfunción familiar.

\section{MÉTODOS}

Se realizó un estudio observacional descriptivo, que tomó como población sujeto de estudio todos los pacientes con edades entre los 18 años y los 65 años que estaban asistiendo a la consulta externa de la clínica CEMIC, en Cartagena, Colombia, durante los meses de septiembre a diciembre de 2018, con diagnóstico CIE 10 en la categoría de esquizofrenia y trastornos del estado de ánimo, que estuviesen recibiendo medicación psiquiátrica al momento de ingresar al estudio, a quienes se les solicitó firmar un consentimiento para participar en la investigación. Fueron excluidos pacientes con deterioro neuro-cognitivo, retraso mental o cociente intelectual menor de 70, déficit en la comunicación verbal, embarazo o lactancia.

El cálculo de la muestra se realizó teniendo en cuenta un nivel de confianza del $95 \%$ un error de $5 \%$ y una proporción esperada de no adherencia entre 30 - 50\% según estudios previos (22)(20), arrojando un total de 198 pacientes con el apoyo del programa Epidat 4.1.

A todos los pacientes se les aplicó un cuestionario creado por los investigadores para indagar características sociodemográficas como edad, sexo, escolaridad, estado civil y ocupación entre otras; además antecedentes de hábitos de consumo de alcohol, tabaco y otras sustancias psicoactivas, 
características del diagnóstico, tipo de diagnóstico, tiempo con el diagnóstico, número de ingresos psiquiátricos; también se indagaron características del tratamiento como el tipo de fármaco usado, la presentación del fármaco y la posología diaria, también se preguntó quién era la persona responsable de administrarles el tratamiento.

Se aplicó la escala APGAR familiar para evaluar el grado de disfunción familiar (23). Además se aplicó la escala de MORISKY GREEN para valorar el cumplimiento de la medicación (24), que consiste en una serie de cuatro preguntas de contraste con respuesta dicotómica (Sí/No), que refleja la conducta del paciente respecto al cumplimiento. Se pretende valorar si el paciente adopta actitudes correctas con relación al tratamiento para su enfermedad; se asume que si las actitudes son incorrectas el paciente es incumplidor. Presenta la ventaja de proporcionar información sobre las causas del incumplimiento. El cuestionario tiene una sensibilidad de 0,81 , una especificidad de 0,44 y una fiabilidad (Cronbach) de 0,61(24) y contiene las siguientes preguntas:

1. ¿Olvida alguna vez tomar los medicamentos para tratar su enfermedad?

2. ¿Toma los medicamentos a las horas indicadas?

3. Cuando se encuentra bien, ¿deja de tomar la medicación?

4. Si alguna vez le sienta mal, ¿deja usted de tomarla?

El paciente es considerado como cumplidor si responde de forma correcta a las cuatro preguntas, es decir, No/Sí/No/No. Es adherente con cuatro respuestas correctas y no adherente menor a cuatro respuestas correctas (24)(25).

\section{Análisis estadístico}

El análisis estadístico descriptivo de variables cualitativas se realizó mediante el cálculo de frecuencias absolutas y relativas, por su parte el de las cuantitativas con medidas de tendencia central tipo Mediana (Me), con sus respectivas medidas de dispersión rango inter-cuartílico (RIC), por el comportamiento no paramétrico de estas variables estimados con la prueba de Kolmogorov-Smirnov.
Para comparar la distribución de las variables cualitativas entre los pacientes con y sin adherencia al tratamiento se utilizaron la prueba Chi2 o el test exacto de Fisher; para comparar las variables cuantitativas se usó la prueba U de Mann Whitney. Un valor de $\mathrm{p}<0,05$ fue considerado como estadísticamente significativo. (EpiInfo)

\section{RESULTADOS}

En total se identificaron 198 pacientes, de los cuales fueron excluidos 38 por presentar limitaciones cognitivas para responder el cuestionario, por lo que se incluyeron 160 pacientes en el estudio.

La mediana de edad fue de 45 años (RIC: 34 - 55), siendo el 56,9\% $(\mathrm{n}=91)$ de sexo femenino, el grado escolar más alto alcanzado más frecuente fue la secundaria completa con $32,5 \%(n=52)$, seguido de secundaria incompleta con $21,3 \%(n=34)$. El estado civil fue soltero en el $50 \%(n=80)$. de los casos y casado en el $24,4 \%(n=39)$. A la pregunta de cuantos hijos tiene el 36,3\% $(\mathrm{n}=58)$ respondió ninguno, el $25 \%(n=40)$ tres o más, el 20,6\% $(n=33)$ dos, y el $18,1 \%(n=29)$ dijo que tenía un hijo. El 81,3\% $(\mathrm{n}=130)$ de los pacientes residían en el área urbana, pertenecían al estrato socioeconómico 1 el 48,8\% $(\mathrm{n}=78)$ y al estrato socioeconómico 2 el $35,6 \%$ $(\mathrm{n}=57)$. El tipo de afiliación al régimen de salud fue al régimen subsidiado en el $91,3 \%(n=146)$. La respuesta más frecuente a la pregunta sobre su ocupación fue desempleada en el $38,8 \% \quad(n=62)$ seguido de trabajo ocasional informal en el $30 \%$ $(n=48)$ y de trabajo regular formal en el $21,3 \%$ $(n=34)$, en menores proporciones se encontraron los pacientes que nunca han trabajado y los jubilados.

Dentro de los antecedentes de consumo en los treinta días previos a la realización de la encuesta se encontró consumo de tabaco en $6,9 \%(n=11)$ de los pacientes, consumo de alcohol en el 6,3\% $(\mathrm{n}=10)$, y consumo de sustancias psicoactivas en $1,9 \%(n=3)$ de ellos.

El 68,1\% $(n=109)$ de los encuestados refirieron conocer su diagnóstico psiquiátrico. El diagnóstico más frecuente con $35,6 \%(n=57)$ fue el trastorno 
afectivo bipolar, seguido de la esquizofrenia con $34,4 \%(n=55)$, y el trastorno depresivo con $22,5 \%$ $(\mathrm{n}=36)$. La mediana de edad al momento del diagnóstico fue de 31 años (RIC: 23 - 45), el tiempo en años con el diagnóstico tuvo una mediana de 6 años (RIC: 2 - 16). No hubo ingresos hospitalarios por causa psiquiátrica en el $29,4 \%(n=47)$ de los casos, mientras que tuvieron tres o más el $27,5 \%$ $(n=44)$, uno el $25,6 \%$ y dos el $17,5 \%(n=28)$ de los pacientes.

En cuanto a las características del tratamiento farmacológico recibido, se encontró a los antipsicóticos atípicos como los más frecuentemente utilizados con 64,4\% ( $n=103)$, seguidos de los estabilizadores del estado de ánimo con 54,4\% $(n=87)$, antidepresivos con $39,4 \%(n=63)$ y las benzodiacepinas con $29,4 \%(n=47)$. La presentación más frecuentemente consumida por los pacientes fue la tableta en el 95\% $(n=152)$ de los casos. La frecuencia del número de medicamentos consumidos diariamente fue de dos a cuatro en el $66,9 \%(n=107)$, seguido de 5 o más en el $23,1 \%$ $(n=37)$ de los casos.

En el 70,6\% ( $\mathrm{n}=113)$ de los casos el responsable de administrar los medicamentos era el propio paciente, $y$ en el $28,1 \%(n=45)$ era un familiar o amigo en. La aplicación del APGAR familiar mostró una frecuencia de familia disfuncional en el $36,3 \%$, siendo esta disfuncionalidad leve en $20,6 \%(n=33)$, moderada en el $5,6 \%(n=9)$, y severa en el $10,0 \%$ $(n=16)$ de los casos. El 55,6\% $(n=89)$ de los encuestados refirieron contar con apoyo de amigos. La escala de Morisky Green mostró no adherencia total al tratamiento de 53,8\% $(\mathrm{n}=86)$, con $56 \%$ $(n=31)$ para el diagnóstico de esquizofrenia, 50,8\% $(n=29)$ para el trastorno afectivo bipolar, y $47,2 \%$ $(\mathrm{n}=17)$ para los trastornos depresivos.

Al comparar la frecuencia de diagnósticos, uso, presentación y frecuencia de fármacos, responsable de administrar tratamiento, funcionalidad familiar y apoyo de amigos estratificado por grupos de pacientes con y sin adherencia al tratamiento, no se observaron diferencias estadísticamente significativas $(\mathrm{p}>0,05)$, (Tabla 1$)$.

\section{DISCUSIÓN}

El control médico ambulatorio de trastornos mentales crónicos y con alto riesgo de recaídas como la esquizofrenia, el trastorno bipolar y la depresión, produce un impacto positivo no solo en quien lo padece sino también en su familia y su comunidad (26).

En el año 2012, la Secretaria de Salud de la ciudad de Cartagena (Colombia) reportó un total de 2922 casos de enfermedades mentales siendo la esquizofrenia la que registró mayor porcentaje (22\%), seguida de la depresión (17.4\%) y el trastorno bipolar se ubicó en el cuarto lugar con un 9.1\% (8). El anterior registro se basó en el diagnóstico clínico realizado en pacientes que asistieron por primera vez a la consulta psiquiátrica, en cambio, nuestro estudio se dirigió a pacientes que ya tenían un historial clínico o que suelen consultar regularmente por lo cual planteamos que esta podría ser la causa de la diferencia en la frecuencia diagnóstica hallada en los resultados.

Debemos tener en cuenta que los pacientes con trastornos mentales que viven en la periferia de los municipios y poblaciones alejadas de Cartagena y requieren control periódico, pudieron quedar por fuera del estudio, puesto que, nuestra población procedía en su mayoría del área urbana. Esto concuerda con el último estudio del Departamento Administrativo Nacional de Estadísticas (DANE), que reporta que el $77.8 \%$ de la población Colombiana vive en cabeceras municipales y solo el $15.1 \%$ vive en la zona rural (27). Es entonces que surge la pregunta si los pacientes con trastornos mentales que viven en el área rural pueden tener acceso a la atención por profesionales especializados en salud mental.

En este estudio el promedio de edad, pertenece a la etapa adulta intermedia. Las personas en esta etapa manifiestan mayor importancia a las recomendaciones del médico ya que a esta edad se alcanza la máxima responsabilidad personal en torno al cuidado de la salud, debido a la mayor vulnerabilidad percibida ante la enfermedad o la 
muerte, a diferencia de la etapa de adolescencia o adultez temprana en la que se perciben menos vulnerables y son más independientes frente a la atención médica (28). Esta variable fue un predictor significativo de la adherencia a la medicación en un estudio realizado en la Universidad Autónoma de Nuevo León México, en pacientes diabéticos (29).

Tabla 1. Comparación de los diagnósticos CIE-10, características del tratamiento y funcionalidad familiar estratificados por adherencia o no adherencia al tratamiento

\begin{tabular}{|c|c|c|c|}
\hline & $\begin{array}{l}\text { Adherencia } \\
\mathrm{N}=74\end{array}$ & $\begin{array}{l}\text { Sin Adherencia } \\
\mathrm{N}=86\end{array}$ & Valor $\mathrm{p}$ \\
\hline \multicolumn{4}{|l|}{ Diagnostico CIE 10} \\
\hline Trastorno Afectivo Bipolar & $28(37,8)$ & $29(33,7)$ & 0,5876 \\
\hline Esquizofrenia & $24(32,4)$ & $31(36,1)$ & 0,6313 \\
\hline Trastorno depresivo & $19(25,7)$ & $17(19,8)$ & 0,3722 \\
\hline Trastorno esquizo afectivo & $3(4,1)$ & $9(10,5)$ & 0,1247 \\
\hline \multicolumn{4}{|c|}{ ¿Qué tratamiento le está formulando el psiquiatra? } \\
\hline Antipsicótico atípico & $44(59,5)$ & $59(68,6)$ & 0,2284 \\
\hline Estabilizador del animo & $43(58,1)$ & $44(51,2)$ & 0,3791 \\
\hline Antidepresivo & $32(43,2)$ & $31(36,1)$ & 0,3529 \\
\hline Benzodiacepinas & $20(27,0)$ & $27(31,4)$ & 0,5452 \\
\hline Antipsicótico típico & $17(23,0)$ & $18(20,9)$ & 0,7553 \\
\hline Anticolinérgicos & $13(17,6)$ & $18(20,9)$ & 0,5915 \\
\hline \multicolumn{4}{|c|}{$\begin{array}{l}\text { Qué presentación de medicamento tiene formulado en este } \\
\text { momento }\end{array}$} \\
\hline Tabletas o capsulas & $70(94,6)$ & $82(95,4)$ & 0,8277 \\
\hline Tabletas y ampollas & $4(5,4)$ & $4(4,6)$ & \\
\hline \multicolumn{4}{|c|}{ ¿Cuántos medicamentos toma durante el día? } \\
\hline Una & $6(8,1)$ & $10(11,6)$ & 0,4593 \\
\hline Dos a cuatro & $50(67,6)$ & $57(66,3)$ & 0,9966 \\
\hline Cinco o más & $18(24,3)$ & $19(22,1)$ & 0,7385 \\
\hline \multicolumn{4}{|l|}{ Responsable del tratamiento } \\
\hline El mismo & $51(68,9)$ & $62(72,1)$ & 0,6602 \\
\hline Familiar/amigo & $23(31,1)$ & $22(25,6)$ & 0,4404 \\
\hline Cuidador & $0(0,0)$ & $2(2,3)$ & 0,4996 \\
\hline Familiar disfuncional & $28(37,8)$ & $30(34,9)$ & 0,6983 \\
\hline Leve & $17(23,0)$ & $16(18,6)$ & 0,4959 \\
\hline Moderada & $5(6,8)$ & $4(4,7)$ & 0,7339 \\
\hline Severa & $6(8,1)$ & $10(11,6)$ & 0,5991 \\
\hline Con soporte de amigos & $44(59,5)$ & $45(52,3)$ & 0,3651 \\
\hline
\end{tabular}

Con respecto al nivel de educación, el $50 \%$ de los pacientes incluidos en este estudio terminaron su educación media y menos de un cuarto cursaron educación superior, equivalente a que actualmente más de la mitad estén desempleados o tengan un trabajo informal. Esto genera que los pacientes y sus familias se encuentren en una situación vulnerable socioeconómicamente, con servicio de salud subsidiado por el Estado. Sin embargo no hubo asociación estadística entre ninguna variable sociodemográfica y el grado de adherencia al tratamiento farmacológico según los hallazgos de un estudio transversal retrospectivo realizado en España con 76 pacientes con trastorno afectivo bipolar con estabilidad clínica (30). 
Es necesario que se aproveche la consulta médica en esta población para informar en que consiste la salud mental y el valor que tiene la adherencia al tratamiento psiquiátrico formulado. La importancia de educar al paciente en cuanto a su patología mental está expuesta en un artículo de revisión del año 2009 (11), en donde una de sus conclusiones es que la educación es una intervención recomendada para mejorar la adherencia a las indicaciones médicas y debe ser un reto a implementarla en los centros de atención en salud mental (31).

Lo anterior podría influir en el resultado de este estudio, en que casi la mitad de los sujetos ha mantenido un adecuado control ambulatorio con dos o menos hospitalizaciones a causa de los planes educativos que se imparten en la clínica a los pacientes en el manejo intra y extrahospitalario.

Los datos de nuestro estudio identifican que más de la mitad de la población no tiene pareja sentimental, que se equilibra, quizás, con familias funcionales y con un adecuado soporte de amigos. La función familiar adecuada es un factor protector para los enfermos y les ayuda a evitar recaídas. El soporte familiar acompaña, apoya, detecta y recibe la información necesaria para la continuidad del cuidado del paciente y se convierte en el canal a través del cual fluye la comunicación con el enfermo (32). En un importante estudio que se realizó con 214 pacientes en un hospital psiquiátrico en la ciudad de Bogotá (10), se efectuó un seguimiento durante un año luego del egreso hospitalario y los resultados arrojaron una alta tasa de rehospitalización temprana, es decir dentro de los tres meses siguientes; los factores que mostraron una fuerte relación estadística, tenían que ver con el soporte social dentro del cual estaba el paciente, el estado civil (soltero o divorciado), el consumo de sustancias psicoactivas y las enfermedades médicas concomitantes.

Otra variable estudiada fue el consumo de sustancias psicoactivas incluyendo alcohol y cigarrillo en los treinta días previos, la cual tuvo un resultado más bajo que lo esperado, teniendo en cuenta que, la literatura médica reporta la frecuente coexistencia de enfermedades mentales con abuso de sustancias psicoactivas, conocido como diagnóstico dual (33). Comparado con los resultados de un artículo de revisión acerca de la adherencia al tratamiento antipsicótico oral donde se examinaron 187 pacientes con enfermedades mentales crónicas que vivían en la comunidad, se encontró que aproximadamente un tercio de la muestra abusaba del alcohol, las drogas ilegales o ambos, durante los seis meses anteriores a la evaluación (34).

En cuanto a la adherencia, en este estudio el porcentaje de pacientes no adherentes al tratamiento farmacológico, incluyendo los tres diagnósticos estudiados, está por debajo de lo hallado en un estudio descriptivo prospectivo realizado en Sao Paulo, Brasil, donde se incluyeron todos los pacientes dados de alta de una unidad de salud mental con un total de 48 sujetos estudiados, reportando un $33 \%$ con diagnóstico de esquizofrenia, $12 \%$ con trastorno afectivo bipolar y $16 \%$ con trastornos del humor depresivos, a los cuales aplicaron el Test de Morisky Green para medir la adherencia según el grado de adhesión en alto y bajo nivel, hallando que el $70,8 \%$ de ellos fueron clasificados con bajo grado de adherencia al tratamiento farmacológico (35). Esta graduación se realizó según el número de respuestas correctas al cuestionario. Esta diferencia de resultados podría deberse a dos factores, el primero a que los sujetos de estudio fueron recién egresados de internación psiquiátrica contrario al nuestro dónde eran pacientes con asistencia voluntaria a control ambulatorio, y el segundo puede ser el tamaño muestral estudiado.

Quizás si analizamos la adherencia según el tipo de diagnóstico: en sujetos con esquizofrenia, los cuales fueron los más adherentes en el grupo estudiado, el resultado se encuentra dentro del amplio rango reportado en la literatura $24 \%$ hasta el $70 \%$ (36) (37) (38). En el trastorno afectivo bipolar la literatura mundial oscila entre $35-50 \%$ (9) (37). En Colombia un estudio pionero de adherencia en trastorno bipolar realizado con 124 pacientes ambulatorios, reportó que el $29.8 \%$ no se adhería a los fármacos de mantenimiento, estadísticamente significativos en 
dicho resultado fueron: antecedente de no adherencia $(39 \%, \mathrm{p}=0,001)$, el hábito de fumar $(47,6 \%, p=0,048)$, mayor disfunción familiar, no contar con familiares que ayuden al paciente en la toma del medicamento $(73 \%, p=0,001)$ (19). El amplio rango de adherencia en estos pacientes puede deberse a las diferencias metodológicas, por ejemplo, en un estudio prospectivo que evaluó la adherencia farmacológica en esquizofrenia y trastorno afectivo bipolar en 200 pacientes midiendo las concentraciones plasmáticas de medicamentos, la tasa de pacientes con leve y bajo cumplimiento fue cercana al $40 \%, y$, al igual que en el presente estudio, el tipo de tratamiento no se asoció con el cumplimiento terapéutico, sin embargo un hallazgo importante fue que la comorbilidad con los trastornos de la personalidad se asoció fuertemente a la ausencia de cumplimiento (39).

En cuanto a la depresión no se encontraron estudios en Colombia que midieran la adherencia a los antidepresivos; sin embargo, en India, un importante estudio transversal de 103 pacientes psiquiátricos con diagnóstico de depresión unipolar reportó un $44 \%$ con deficiente adherencia farmacológica, agregado a un $26 \%$ con una "justa" adherencia, la cual agrupan como no-adherente. No fue significativo el tipo de medicamento, pero el estudio resalta el valor estadísticamente significativo de la polifarmacia oral $(p<0.043)(16)$. La polifarmacia se define simplemente como el uso de múltiples medicamentos por un paciente, aunque el número mínimo preciso de los medicamentos utilizados para definir "polifarmacia" es variable. Esta se describe numéricamente como cinco o más medicamentos recetados en cualquier momento, incluyendo los medicamentos sin formula médica o los suplementos herbales (40). Contrastado con los resultados de nuestro estudio la cantidad de medicamentos psiquiátricos al día fue entre dos $\mathrm{y}$ cuatro, sin cuantificar otros medicamentos para enfermedades comórbidas.

Es de resaltar que este estudio mostró que casi todos los pacientes tienen prescritos medicamentos orales ya sean tabletas o cápsulas. Una alternativa disponible ante el consumo excesivo de medicamentos orales en estos pacientes podría ser el uso de medicamentos inyectables de larga acción (41).

Creemos que en el presente estudio no se halló relación estadísticamente significativa entre las variables estudiadas y la adherencia al tratamiento farmacológico debido por un lado al tamaño muestral estudiado y por otro, a la disparidad metodológica hallada en los artículos de referencia, ya que no hay un consenso metodológico para la medición objetiva de la adherencia (conteo de tabletas, diferentes test de autoevaluación, etc) (42), además de las diferencias sociodemográficas de las poblaciones en estudio como la edad, el tiempo de egreso hospitalario, la edad de diagnóstico o el tiempo de tratamiento. Otra limitación del presente estudio fue que no se utilizó ninguna herramienta estructurada para el diagnóstico del trastorno bipolar, la esquizofrenia o la depresión, sino, la historia clínica proporcionada por cada paciente, ni se aplicó un estudio piloto previo para moldear los cuestionarios aplicados a la población.

Un sesgo que identificamos conforme al resultado de la baja frecuencia de no adherencia al tratamiento puede deberse a que la población estudiada asiste frecuentemente a los controles programados por psiquiatría, excluyendo aquellos que no se adhieren a las recomendaciones ambulatorias en citas por primera vez, egresos hospitalarios o remisiones de médico general u otro médico especialista.

Dentro de las fortalezas, se encuentran el cálculo estadístico de la muestra, la utilización de cuestionarios validados en nuestro país e idioma, además de ser pionero en el estudio epidemiológico como una aproximación a la población que a diario nos encontramos los psiquiatras en nuestra práctica clínica.

Más de la mitad de pacientes tratados ambulatoriamente en la clínica del estudio pertenecen a los diagnósticos de esquizofrenia y trastorno afectivo bipolar, y según las características sociodemográficas hacen parte de un grupo vulnerable con condiciones socioeconómicas 
desfavorables, pero a pesar de eso las tasas de hospitalización, el abandono al tratamiento farmacológico, y la disfuncion familiar son estadisticamente bajos, comparado con los eprotado por otros estudios. Se resalta la necesidad de realizar más estudios epidemiológicos donde se logre caracterizar los pacientes hospitalizados, determinando la prevalencia de rehospitalizaciones e identificar los factores personales de la población como el estigma a la enfermedad mental, las características de la enfermedad, la alianza terapeutica médico-paciente, y la accesibilidad a los servicios de dispensación de medicamentos, entre otros.

AGRADECIMIENTOS: A la Clínica Psiquiátrica CEMIC, por permitir la realización del estudio $\mathrm{y}$ estar prestos en la realización del mismo.

CONFLICTOS DE INTERESES: ninguno que declarar.

FINANCIACION: recursos propios de los autores.

\section{REFERENCIAS}

1. Bessant, M., Ekers D. Common mental health disorders. Nursing Times [en línea]. 2011. [fecha de acceso 20 mayo de 2019]; No.107 URL disponible en: http://www.ncbi.nlm.nih.gov/pubmed/21827090

2. Posada J. A. La salud mental en Colombia. Biomédica [en línea]. 2013. [fecha de acceso 20 mayo de 2019]; No. 33 URL disponible en: http://www.scielo.org.co/scielo.php?script=sci_arttext\&pid $=$ S0120-41572013000400001\&lng $=$ en

3. Bhugra, D.The Global Prevalence of Schizophrenia. PLoS Medicine [en línea]. 2005. [fecha de acceso 3 diciembre de 2018] URL disponible en: https://doi.org/10.1371/journal.pmed.0020151

4. Terrence A. Ketter M. Diagnostic Features, Prevalence, and Impact of Bipolar Disorder. J Clin Psychiatry. 2010;71(6):14

5. NICE guidelines. Bipolar disorder: assessment and management [en línea]. National Institute for Health and Care Excellence;2014. [fecha de acceso 16 febrero de 2019]. URL disponible en: https:/www.nice.org.uk/guidance/cg185/chapter/1recommendations\#how-to-use-medication
6. Andrade L, Berglund P et al. The epidemiology of major depressive episodes: results from the International Consortium of Psychiatric Epidemiology (ICPE) Surveys. Int J Methods Psychiatr Res. 2002;12(1)

7. Ministerio de Salud y Protección social. Guía Metodológica para el Observatorio Nacional de Salud Mental [en línea]. 2017. [fecha de acceso 16 febrero de 2019] No.47 URL disponible en: https://www.minsalud.gov.co/sites/rid/Lists/BibliotecaDigi tal/RIDE/VS/ED/GCFI/guia-ross-salud-mental.pdf

8. Perfil epidemiológico de Cartagena 2012 [en línea]. Cartagena de Indias. Departamento Administrativo Distrital de Salud- DADIS; 2013. [Fecha de acceso 20 noviembre 2018]. URL disponible en: https://www.minsalud.gov.co/Documentos $\% 20 \mathrm{y} \% 20$ Publi caciones/ASIS\%20Cartagena\%202012.pdf

9. Chakrabarti S. Treatment-adherence in bipolar disorder: A patient-centred approach. World J Psychiatry. 2016;6(4):399-409

10. Sánchez R, Jaramillo L, Herazo M, Factores asociados a rehospitalización temprana en psiquiatría. Biomédica. 2013;33:276-82

11. Julius RJ, Dubin WR, Medication Adherence: A Review of the Literature and Implications for Clinical Practice. J Psychiatr Pr. 2009;15(1):34-44

12. Vieta E, Azorin J, Bauer M, Frangou S, Perugi G, Martinez G, et al. Psychiatrists' perceptions of potential reasons for non- and partial adherence to medication: Results of a survey in bipolar disorder from eight European countries. J Affect Disord. 2012;143(1-3):125-30.

13. Adams J, Scott J. Predicting medication adherence in severe mental disorders. Acta Psychiatr Scand. 2000;119-24

14. Keck P, Mcelro $\mathrm{Y}$ et al. Factors associated with pharmacologic non-compliance in patients with mania. $\mathrm{J}$ Clin Psychiatry. 1996; 57: 292 2297

15. Myers E, Branthwaite A. Outpatient compliance with antidepressant medication. $\mathrm{Br} \mathrm{J}$ Psychiatry. 1992;(160):83 \pm 86

16. Sriharsha P. Alekhya B. Shivanandh et al. Treatment and disease related factors affecting nonadherence among patients on long term therapy of antiderpressants. Indo Am J Pharm Res. 2015;5(03)

17. Adherence to Long-term Therapies policy for action [en linea]. Switzerland: World Health Organization; 2001.[fecha de acceso 18 de noviembre de 2018]. URL disponible

en: 
https://www.who.int/chp/knowledge/publications/adherenc e_report/en/

18. Osterberg L, Blaschke T. Adherence to Medication. n engl j med. 2005; 353;(5):487-97

19. Elena E, Cely P, Fierro M. Prevalencia y factores asociados a la no adherencia en el tratamiento farmacológico de mantenimiento en adultos con trastorno afectivo bipolar. Rev Colomb Psiquiat. 2011;40(1):85-98

20. Lingam R, Treatment SJ. Treatment non-adherence in affective disorders. Acta Psychiatr Scand. 2002;105(10):164-172

21. Bollini P, Pampallona S, Kupelnick B, Tibaldi G, Munizza C. Improving compliance in depression: a systematic review of narrative reviews. Journal of Clinical Pharmacy and Therapeutics. 2006;(31):253-260

22. Clatworthy J, Bowskill R, Rank T, Parham R. Adherence to medication in bipolar disorder: a qualitative study exploring the role of patients' beliefs about the condition and its treatment. 2007;(5):656-64

23. Suarez M, Apgar Familiar : Una Herramienta Para Detectar Disfunción Familiar. Rev. Médica La Paz [en línea]. 2014. [fecha de acceso 10 octubre de 2018]; No.1 URL Disponible en: http://www.scielo.org.bo/pdf/rmcmlp/v20n1/v20n1_a10.pd $\underline{f}$

24. Morisky D, Ang A, Krousel-wood M, Ward HJ. Predictive Validity of a Medication Adherence Measure in an Outpatient Setting. J Clin hypertens. 2008;10(5):348-354

25. Nogués, X. Sorli, M., Villar, L. J. Instrumentos de medida de adherencia al tratamiento. Anales de Medicina Interna. (2007);(24):138-141

26. Sajatovic M, Velligan D, Weiden P, Valenstein M, Ogedegbe G. Measurement of Psychiatric Treatment Adherence. J Psychosom Res.2010;69(6):591-599

27. Censo nacional de población y vivienda [en línea]. Colombia: Departamento Administrativo Nacional de Estadística DANE; 2018. [fecha de acceso 15 mayo de 2019]. URL disponible en: http://www.dane.gov.co/index.php/estadisticas-portema/demografia-y-poblacion/censo-nacional-depoblacion-y-vivenda-2018

28. Devoe J.E, Wallace L.S, Fryer G.E. Patient age influences perceptions about health care communication. Family Medicine.2009;41(2):126-133

29. De la Rubia J. M, Cerda M.T. Predictores psicosociales de adherencia a la medicación en pacientes con diabetes tipo 2. Revista Iberoamericana de Psicología y Salud. 2015;6(1):19-27.

30. Navarro S, Rodríguez F, Acosta FJ. Variables asociadas a la no adherencia en pacientes con trastorno bipolar en estabilidad clínica. Actas Esp Psiquiatr. 2016;44(5):157-165

31. Feria I. Intervenciones educativas para enfermos de salud mental con medicación psicotrópica. Enfermería Clínica [en línea]. 2008. [fecha de acceso 15 mayo de 2019]:No.3 URL disponible en: https://doi.org/10.1016/s1130-8621(08)70722-8

32. Patiño D.C, Andrea V, Guzmán Z, Victoria L, Bernal L, Ángela L, et al. Family support received through psychiatric treatment by patients with a diagnosis of mental illness. Revista Colombiana de Ciencias Sociales. 2012;3(2):243-58

33. Roncero C, Barral C, Casas M. Protocolos de intervención en patología dual [en línea]. Barcelona: EdikaMed;2016. [fecha de acceso 13 mayo de 2019] URL disponible en: https://patologiadual.es/protocolos-deintervencion/

34. Velligan D, Lam Y, Glahn D, Barrett J, Maples N, Ereshefsky L et al. Defining and Assessing Adherence to Oral Antipsychotics: A Review of the Literature. Schizophrenia Bulletin. 2006;32(4):724-742

35. Cardoso L, Inocenti A, Frari S, Marques B, Braga R. Grado de adhesión y conocimiento sobre tratamiento psicofarmacológico entre pacientes egresados de internación psiquiátrica. Rev Latino-Am Enfermagem [en línea]. 2011. [fecha de acceso 13 mayo de 2019]; No 5. URL disponible

http://www.scielo.br/pdf/rlae/v19n5/es_12.pdf

36. Gilmer T. Dolder C, Lacro J, Folsom D, Lindamer L, Garcia, $\mathrm{P}$ et al. Adherence to Treatment with Antipsychotic Medication and Health Care Costs among Medicaid Beneficiaries with Schizophrenia. American Journal of Psychiatry. 2004;161(4):692-699

37. Lieberman J. A. Effectiveness of antipsychotic drugs in patients with chronic schizophrenia. The Journal of Clinical Psychiatry [en linea]. 2009. [fecha de acceso 13 mayo de 2019]; No. 2 URL disponible en: https://doi.org/10.4088/jcp.0207e04

38. Kim J, Ozzoude M, Nakajima S, Shah P, Caravaggio $\mathrm{F}$, Iwata $\mathrm{Y}$, et at. Insight and medication adherence in schizophrenia: An analysis of the CATIE data. Neuropharmacology. [en linea]. 2019. [fecha de acceso 13 mayo de 2019]. URL disponible en: https://doi.org/10.1016/j.neuropharm.2019.05.011 
39. Colom F, Vieta E, Martinez A, Reinares M, Benabarre A, Gasto C. Clinical factors associated with treatment noncompliance in euthymic bipolar patients. Journal of Clinical Psychiatry. 2000;61(8):549-555.

40. Castro J. A, Orozco J. P, Marín D. S. Polifarmacia y prescripción de medicamentos potencialmente no apropiados en ancianos. Rev. Méd. Risaralda. 2016;22(1):52-57
41. Rimal B. Bera M. Patient Outcomes Within Schizophrenia Treatment: A Look at the Role of LongActing Injectable Antipsychotics. J Clin Psychiatry. 2014;75(2):30-33

42. Velligan D.I, Weiden P.J et al. The expert consensus guideline series: adherence problems in patients with serious and persistent mental illness. J Clin Psychiatry. 2009;70:1-46 